\title{
Sensitivity Analysis of Decision Making Under Dependent Uncertainties Using Copulas
}

\begin{abstract}
Many important decision and risk analysis problems are complicated by dependencies between input variables. In such cases, standard one-variable-at-a-time sensitivity analysis methods are typically eschewed in favor of fully probabilistic, or $n$-way, analysis techniques which simultaneously vary all $n$ input variables and capture their interdependencies. Unfortunately, much of the intuition provided by one-way sensitivity analysis may not be available in fully probabilistic methods because it is difficult or impossible to isolate the marginal effects of the individual variables. In this paper, we present a dependence-adjusted approach for identifying and analyzing the impact of the input variables in a model through the use of probabilistic sensitivity analysis based on copulas. This approach provides insights about the influence of the input variables and the dependence relationships between the input variables. One contribution of this approach is that it facilitates assessment of the relative marginal influence of variables for the purpose of determining which variables should be modeled in applications where computational efficiency is a concern, such as in decision tree analysis of large scale problems. In addition, we also investigate the sensitivity of a model to the magnitude of correlations in the inputs.
\end{abstract}

Keywords: Decision Analysis, Sensitivity Analysis, Correlations, Dependence, Copulas 


\section{Sensitivity Analysis of Decision Making Under Dependent Uncertainties Using Copulas}

\section{Introduction}

Decision makers using mathematical models to make decisions are often uncertain about the values of one or more model input parameters. Sensitivity analysis is concerned with understanding how changes in the model inputs influence the outputs. The US Office for Management and Budget prescribes that "Sensitivity analysis is generally considered a minimum, necessary component of a quality risk assessment report.” (OMB Proposed Risk Assessment Bulletin, 2006)

The input quantities in a decision model must be estimated using methods that range from the analysis of historical data to intelligent guesses. The base case values for variables are often not known with precision, and most common sensitivity analysis methods assume independence between model inputs. One of the most frequently used approaches is a one-way sensitivity analysis displayed in a Tornado diagram which determines the influence of each input variable by the degree to which the objective function changes as that variable is varied while all other input variables are held fixed (see discussions in Clemen and Reilly 2000; Saltelli and Annoni 2010; Hazen 2014). Although this approach is sometimes extended to simultaneously analyze two variables at a time, the obvious disadvantages are that it measures the influence of only one (or two) variable(s) and it assumes independence among the input variables. Unfortunately, inputs are often correlated in real world problems, and there are situations when such dependencies could affect the sensitivity analysis results.

The need to conduct sensitivity analysis for problems with dependent input variables has led to the development of fully probabilistic, or $n$-way, methods that simultaneously vary all $n$ variables in a problem. In a probabilistic sensitivity analysis, the analyst assigns probability distributions to uncertain input parameters and estimates the risk profile of the output due to these uncertainties, or alternately, the expected value of perfect information may be used as a sensitivity measure of robustness.

Developments in probabilistic sensitivity analysis approaches include, among others, correlation-based, variance-based, expected value of perfect information (EVPI)-based, and 
output distribution (both density and cumulative) differencing methods (c.f., Wagner 1995; Felli and Hazen 1998; Borgonovo and Smith 2011; Baucells and Borgonovo 2013). Correlation-based approaches calculate correlations between the model input and output either from the Pearson linear product moment correlation, or from the standardized regression coefficient (Saltelli and Marivoet 1990; Campolongo and Saltelli 1997). Variance-based approaches calculate each input variable's contribution to the total output variance as sensitivity measures by holding all other inputs constant (Iman and Hora 1990). Expected value of information (EVPI)-based sensitivity assessments (Felli and Hazen 1998; Felli and Hazen 2004; Hazen 2014) such as the Javelin diagrams (Felli and Hazen 2004) report the EVPI of input variables. Output distribution differencing methods measure input sensitivity by comparing the prior (unconditional) and posterior output (conditional) distributions with and without the uncertainty (c.f. Borgonovo 2007; Baucells and Borgonovo 2013; Wei et al. 2014). We refer the interested readers to Oakley and O'Hagan (2004), Baucells and Borgonovo (2013) and Borgonovo (2013) for excellent reviews of these methods.

A few important challenges exist when modeling probabilistic sensitivity analysis. First, dependence relationships among input variables must be included. Of the $n$-way approaches discussed above, the correlation-based, variance-based, density-based, and Javelin diagram methods do not handle dependence well and may require additional information, e.g., a full specification of input probability distributions or of utilities, as discussed in Baucells and Borgonovo (2013). Some recent developments in the literature (c.f. Mara and Tarantola 2012; Kucherenko et al. 2012; Baucells and Borgonovo 2013) show how to incorporate correlations in the variance-based or cumulative distribution-based methods when there is a closed form conditional distribution in the case of a multivariate normal distribution or under the assumption that only the first-order conditional moment characterizes the dependences between the inputs. It is challenging, however, to incorporate correlations in these methods when the closed form conditional distribution is not available or when the marginals are from different distributions.

The second challenge in probabilistic sensitivity analysis is to identify the marginal effects of input variables on decisions. The $n$-way methods mentioned above do provide some insights into the sensitivity of input variables with higher moment information (e.g., variance-based 
methods) or averaged distribution information (e.g., density or cumulative distributions-based methods). However, this information may not be relevant for identifying the input variables that may cause the choice among decision alternatives to switch as they change over reasonable ranges. For instance, Baucells and Borgonovo (2013) measure how much an input influences the output by averaging the distance between the unconditional and the conditional (on that input) distributions of the output. The most influential uncertainty under the Baucells and Borgonovo (2013) measure will on average deviate from the CDF the most, but this information may not change the decision.

Finally, a third challenge with fully probabilistic $n$-way methods is to present the results of an analysis in a manner that is as intuitive as possible. Although the methods discussed above may depict the overall joint sensitivity of an output to changes in the inputs to a model, they may not show the impacts of changes in the individual input variables on the output of the model. This is because most of the fully probabilistic methods lack a graphical means for presenting the results. Because of this, their sensitivity analysis of dependence among the uncertainties may not be useful for practitioners in decision and risk analysis (cf. Kucherenko et al. 2012; Wei et al. 2014). One noticeable exception is the Javelin diagram developed by Felli and Hazen (2004) who introduce the Javelin diagram as a graphical tool to display the EVPI associated with individual input parameters. However, the Javelin diagram is intended to address the post hoc robustness of decisions to parameter estimates in the defensible stage (Howard 1983) after the analysis is complete (Felli and Hazen 2004), instead of being used in the formation stage of a decision analysis to guide model development which is the focus of this paper. In addition, the Javelin diagram is not designed to directly model the correlations between input parameters.

In this paper, we present a correlation-adjusted sensitivity analysis method based on the use of copulas that addresses the issues above. First, our method incorporates correlations while also isolating the marginal effects of each input variable. Second, our method can be used in the model formulation stage of decision analysis applications. It assists the decision maker with information on whether or not the variation of an input can produce a change in the optimal decision policy. Third, our method provides intuitive graphical output information to facilitate the interpretation of results. 
A copula function links univariate marginal distributions with their multivariate joint distribution and allows flexibility in modeling different dependence relationships through the choice of a specific copula function. As such, copulas provide a convenient way to express multivariate distributions and to model dependence. For an introduction to the theory of copulas and their different families, the reader may refer to Nelsen (2006), Clemen and Reilly (1999) and Avramidis et al., (2009). Wang and Dyer (2012) provide a detailed discussion and examples of the calculations of the dependent uniform variables using a variety of copulas.

Our approach results from a key observation about the properties of copulas. A copula allows a joint distribution of random variables to be constructed from two independent components: the copula and independent marginal distributions. In this structure, the co-movements of these marginal variables must be governed by the dependence relationships since the variables are "coupled together" through the copula function. In our approach, the sensitivity of an input variable is assessed by varying it conditioned on the base values of the other input variables. Therefore, the marginal sensitivity of the model output to changes in each input variable, including all conditional relationships with that variable, can be calculated. With this information, a decision maker can then determine which variables should be carefully assessed, and which variables can be excluded from a probabilistic analysis without a significant loss of accuracy or a resulting change in the decision policy. It can also be difficult to estimate correlations between input variables (c.f. Bornert et al., 2009) and the proposed approach facilitates sensitivity analysis on correlations in decision and risk analysis problems. We believe that this is the first work to address this issue.

The remainder of this paper is organized as follows: Section 2 motivates our new approach with a simple example that allows an analytical solution to the sensitivity analysis using a copula and a comparison with the standard and $n$-way sensitivity analysis approaches. Section 3 provides an explanation of the copula-based approach to sensitivity analysis for more general problems, and shows the advantages of this new approach with an example. Section 4 provides a summary of the work and discusses its limitations. 


\section{Discussion of Sensitivity Analysis Methods Using a Simple Example}

The main purpose of this paper is to provide insights about the isolated marginal influence of the input variables and the dependence relationships between the input variables. Therefore, we will compare our copula approach with the standard one-way sensitivity analysis of the inputs to a model where each input is implicitly assumed to be statistically independent of the other input variables, and the relevant model output is evaluated for the high and the low values for each input. We will also compare our approach with the $n$-way sensitivity analysis whereby each input variable is specified as a probability distribution that may be correlated with other input distributions, and these distributions are simultaneously allowed to vary using Monte Carlo simulation.

Our first example problem is a simple two asset portfolio, and we use it to demonstrate how dependence between input variables can affect sensitivity analysis results and to show how copulas can be used as an alternative method for incorporating dependency. Let $X_{1}$ and $X_{2}$ be bivariate normal random returns of the two assets, with $X_{1} \sim N\left(3 \%, 1 \%^{2}\right)$ representing a relatively safe bond investment and $X_{2} \sim N\left(15 \%, 20 \%{ }^{2}\right)$ representing a risky investment, and these two investments are correlated with $\rho=0.9$. Assume the investor allocates equal weights in the portfolio; $w_{1}=50 \%$ to asset 1 and $w_{2}=50 \%$ to asset 2 . In this case, the portfolio return is

$$
X_{P}=w_{1} X_{1}+w_{2} X_{2}=0.5 X_{1}+0.5 X_{2}
$$

with mean $9.0 \%$. The objective of a sensitivity analysis is to determine which of these two investments has the most influence on the portfolio return. Intuition would suggest the variance of the portfolio returns should be driven by asset 2, due to its higher standard deviation of returns.

Using a standard one-way sensitivity analysis where we vary $X_{1}$ independently by $\pm 3 \sigma_{1}$, we have the following range for the portfolio return $X_{P} \mid\left(X_{2}=\mu_{2}\right)$ :

$$
\left\{w_{2} \mu_{2}+w_{1}\left(\mu_{1}-3 \sigma_{1}\right), w_{2} \mu_{2}+w_{1}\left(\mu_{1}+3 \sigma_{1}\right)\right\}=\{7.5 \%, 10.5 \%\}
$$

Similarly, we can calculate the range of values for the portfolio return based on variations for $X_{P} \mid\left(X_{1}=\mu_{1}\right)$. The results for minimum and maximum output values due to the change in each 
input variable, which are summarized in Panel A in Table 1 and shown graphically with the bars labeled $X_{1}$ (one-way) and $X_{2}$ (one way) in Figure 1, indicate that the portfolio return is more sensitive to the changes in the return of asset 2 . However, since a one-way sensitivity analysis does not account for correlation between the input variables, the decision maker would be unaware of the impact of the dependence on the sensitivity analysis that is captured by the copula approach.

\section{[Insert Table 1 Here]}

\section{[Insert Figure 1 Here]}

The portfolio returns in this example can be represented with a bivariate normal copula where both marginals are normally distributed. That is,

$C_{N}\left(F_{1}\left(X_{1}\right), F_{2}\left(X_{2}\right)\right)=C_{N}\left(u_{1}, u_{2}\right)$

where $F_{1}\left(X_{1}\right)$ and $F_{2}\left(X_{2}\right)$ denote the marginal distributions of $X_{1}$ and $X_{2}$ respectively and $C_{N}$ is a bivariate normal copula.

We first hold $X_{2}$ at its mean value $\mu_{2}$ (or equivalently, $u_{2}=F_{2}\left(\mu_{2}\right)=0.5$ ), and vary $X_{1}$ (or equivalently, $\left.u_{1}=F_{1}\left(X_{1}\right)\right)$ independently. Then we can calculate the conditional uniform variable

$u_{1} \mid u_{2}=\Phi\left(\rho \Phi^{-1}\left(u_{2}\right)+\sqrt{1-\rho^{2}} \Phi^{-1}\left(u_{1}\right)\right)=\Phi\left(\sqrt{1-\rho^{2}} \Phi^{-1}\left(u_{1}\right)\right)$. Next

$X_{1} \mid\left(X_{2}=\mu_{2}\right)=F_{1}^{-1}\left(u_{1} \mid u_{2}\right)=\mu_{1}+\sigma_{1} \sqrt{1-\rho^{2}} \Phi^{-1}\left(u_{1}\right)=\mu_{1}+\sigma_{1} \sqrt{1-\rho^{2}} z, z \sim N(0,1)$.

Using the parameter values from our example, $X_{1} \mid\left(X_{2}=\mu_{2}\right) \sim N\left(3 \%, 0.436 \%{ }^{2}\right)$ and varying $X_{1}$ by $\pm 3 \sigma_{1}$, we can calculate the range of values for the portfolio, $X_{P} \mid\left(X_{2}=\mu_{2}\right)$ :

as

$\left\{w_{2} \mu_{2}+w_{1}\left(\mu_{1}-3 \sigma_{1} \sqrt{\left(1-\rho^{2}\right)}\right), w_{2} \mu_{2}+w_{1}\left(\mu_{1}+3 \sigma_{1} \sqrt{\left(1-\rho^{2}\right)}\right)\right\}$

$=\{8.346 \%, 9.654 \%\}$ 
We similarly calculate the range of changes in the portfolio return for $X_{2} \mid\left(X_{1}=\mu_{1}\right)$ and summarize the results in Panel B of Table 1 and with the bars labeled $X_{1}$ (copula) and $X_{2}$ (copula) in Figure 1. Note that these same formulas for the conditional probability distributions of $X_{1}$ and $X_{2}$ can be obtained for the case of the bivariate normal distribution without the use of the copula, but that will not be true in the more general case that we introduce in the next section.

These results show that the portfolio returns are more sensitive to variations in the returns of asset 2 using the copula approach, but this effect is less pronounced than in the case of the oneway sensitivity results, as the standard one-way sensitivity analysis ignores the correlation and hence mistakenly exaggerates the portfolio variation. Intuitively, accounting for the strong positive correlation effectively places a constraint on the range of variation of the sensitivity variable $\left(X_{1}\right.$ or $\left.X_{2}\right)$ when the other variable $\left(X_{2}\right.$ or $X_{1}$, respectively) is fixed at its mean.

We also conduct an $n$-way (2-way in this example) sensitivity analysis which varies both $X_{1}$ and $X_{2}$ simultaneously, while also modeling the dependency. In this case, the returns for $X_{2}$ are

$X_{1} \mid\left(X_{2}=x_{2}\right) \sim N\left(\mu_{1}+\rho \frac{\sigma_{1}}{\sigma_{2}}\left(x_{2}-\mu_{2}\right), \sigma_{1}^{2}\left(1-\rho^{2}\right)\right)$,

and the portfolio returns are

$X_{P}\left|\left(X_{2}=x_{2}\right)=w_{2} x_{2}+w_{1} X_{1}\right|\left(X_{2}=x_{2}\right)$.

If we vary $X_{1}$ by $\pm 3 \sigma_{1}$ while simultaneously varying $X_{2}$ by $\pm 3 \sigma_{2}$, the minimum and maximum returns for the portfolio are:

$\operatorname{Min}\left\{X_{P} \mid\left(X_{2}=x_{2}\right)\right\}=w_{2}\left(\mu_{2}-3 \sigma_{2}\right)+w_{1}\left(\mu_{1}+\rho \frac{\sigma_{1}}{\sigma_{2}}\left(\mu_{2}-3 \sigma_{2}-\mu_{2}\right)-3 \sigma_{1} \sqrt{\left(1-\rho^{2}\right)}\right)=$ $-23.00 \%$

$\operatorname{Max}\left\{X_{P} \mid\left(X_{2}=x_{2}\right)\right\}=w_{2}\left(\mu_{2}+3 \sigma_{2}\right)+w_{1}\left(\mu_{1}+\rho \frac{\sigma_{1}}{\sigma_{2}}\left(\mu_{2}+3 \sigma_{2}-\mu_{2}\right)+3 \sigma_{1} \sqrt{\left(1-\rho^{2}\right)}\right)$ $=41.00 \%$

We similarly calculate the minimum and maximum for $X_{2} \mid\left(X_{1}=x_{1}\right)$ and summarize the results in Panel $\mathrm{C}$ of Table 1 and graphically with the bars labeled $X_{1}$ (two-way) and $X_{2}$ (two- 
way) in Figure 1. These results again show that the portfolio returns are more sensitive to variations in the returns of asset 2 , but the sensitivity to varying the returns of asset 1 is also now nearly as significant, due to the correlation between returns. Both stocks can appear to have about the same impact on the variability of the returns of the portfolio, because each is being simultaneously varied over its entire range, and so the impacts of one versus the other are more difficult to distinguish compared to the results of the copula analysis.

In summary, the standard one-way sensitivity analysis ignores the correlation between the two variables; the 2-way sensitivity analysis makes it difficult to gauge the relative contributions of the individual variables to the variance of the portfolio. In contrast, our approach assesses the marginal effect of each uncertainty by varying it conditioned on the base values of the other input variables through the use of copula. The portfolio example above is the special case of a bivariate normal copula where a closed form conditional distribution is available. More generally, in a model with $n$ correlated input random variables $X_{1}, X_{2}, \ldots, X_{n}$, the sensitivity of $X_{i}$ would be determined by varying it over its conditional range of $X_{i} \mid\left(X_{\sim i}=\mu_{\sim i}\right)$ as "implied" by the dependency while all other input variables $X_{\sim i}$ are held fixed at their base values $\mu_{\sim i}$. By contrast, with an $n$-way sensitivity analysis, the sensitivity of $X_{i}$ would be determined by varying $X_{i}$ while also allowing $X_{\sim i}$ to vary fully under the specified correlations.

Next we will present the general case of the copula approach where the conditional distribution may not be mathematically tractable.

\section{The Copula Approach for the General Case}

\subsection{The Eagle Airlines Example}

We motivate our discussion using a familiar problem from the literature, the Eagle Airline example (Reilly, 2000; Clemen and Reilly, 1999, 2000), which involves multiple correlated uncertainties. This example has been used in several instances in the decision and risk analysis literature (Clemen and Reilly 2000; Reilly, 2000; Clemen and Reilly, 1999; Wang and Dyer, 2012; and Montiel and Bickel, 2012). 
In this hypothetical decision analysis problem, Dick Carothers, the owner of Eagle Airlines, is deciding whether to invest his profits of $\$ 52,000$ in a money market fund which is expected to deliver an $8 \%$ return or to expand his fleet with the purchase of a second-hand aircraft; hereafter we refer to these alternatives as "Money Market" and "Expand". The annual profit for the latter alternative would be the total annual revenues minus the total annual cost:

Profit $=$ Total Revenue - Total Cost

where,

Total Revenue $=$ Charter Ratio $\times$ Hours Flown $\times$ Charter Price $+(1-$ Charter Ratio $) \times$ Hours Flown $\times$ Capacity $\times$ Number of Seats $\times$ Price Level

Total Cost $=$ Hours Flown $\times$ Operating Cost + Insurance + Purchase Price $\times$ Percentage Financed $\times$ Interest Rate

Charter Price $=3.25 \times$ Price Level, and Number of Seats $=5$.

Sensitivity analyses are typically based on the specification of a base or reference case and of a range of values (bounds) for each input to an analytical model. These values may be obtained from historical data, analytical forecasts, or subjective judgments. Table 2 provides the estimated base case values, reasonable upper $\left(90^{\text {th }}\right.$ percentile) and lower $\left(10^{\text {th }}\right.$ percentile) bounds, and break-even values for the input variables, where we interpret "break-even" for this example to be a value of an input which results in the corresponding value of Profit $=0$ given the remaining inputs are at their base values.

[Insert Table 2 Here]

The "Base" column in Table 2 provides Carothers' initial estimates regarding the 10 input variables. We can use these base values to estimate an annual profit of $\$ 9,975$, which is $\$ 5,775$ more than the estimate of $\$ 4,200(\$ 52,000 \times 8 \%)$ for the money market investment. The deterministic model therefore indicates that Carothers should expand his fleet. However, some of the inputs are highly uncertain and could lower the profit below the $\$ 4,200$ benchmark. 


\subsection{The General Copula-Based Sensitivity Approach}

Consider a random vector $X=\left(X_{1}, \ldots, X_{n}\right)$ with $F_{i}\left(X_{i}\right)$ denoting the marginal distribution of each $X_{i}$. Sklar (1959) showed that the joint distribution $F(X)$ can be expressed as a copula function $C$ associated with the $F_{i}\left(X_{i}\right)$, i.e.,

$F(X)=F\left(X_{1}, \ldots, X_{n}\right)=C\left(F_{1}\left(X_{1}\right), \ldots, F_{n}\left(X_{n}\right)\right)$

The copula function $C$ is itself a distribution function for uniform random variables since the marginal cumulative distribution functions (CDFs) are standard uniform distributions, i.e.,

$C\left(u_{1}, \ldots, u_{n}\right)=F\left(F_{1}^{-1}\left(u_{1}\right), \ldots, F_{n}^{-1}\left(u_{n}\right)\right)$.

An important property of copula functions is that they allow the separation of the marginal distributions from the dependence structure; the dependence structure among the random variables is independent of the choice of the marginal distributions. This facilitates the modeling of dependent multivariate uncertainties by using the marginal distributions $F_{i}\left(X_{i}\right)$ and the copula function in a Monte Carlo simulation (e.g., Embrechts et al., 1999; Kousky and Cooke, 2009; Cherubini et al., 2004; Biller, 2009; Kucherenko et al. 2012; Wei et al. 2014) or a decision tree (Clemen and Reilly, 1999; Wang and Dyer, 2012; Wang, Dyer and Butler, 2015; Wang, Dyer and Hahn 2015) to generate multivariate joint distributions and their discrete approximations.

In what follows, we outline the main concepts of the procedure for our proposed copula based approach to dependent sensitivity analysis. We then illustrate these concepts by applying them to the Eagle Airlines example.

\section{Step 1 - Define the Payoff Functions}

This first step is common to all approaches to sensitivity analysis. Suppose the decision maker has assessed each marginal distribution function $F_{i}\left(X_{i}\right)$ and must select an alternative $a_{j}$ from a set of $j=1, \ldots, m$ feasible alternatives. The value $v\left(a_{j}\right)$ of each alternative is a function of the ultimate true state of nature, which is random multivariate vector $X=\left(X_{1}, \ldots, X_{n}\right)$ with joint distribution

$F(X)=F\left(X_{1}, \ldots, X_{n}\right)=C\left(F_{1}\left(X_{1}\right), \ldots, F_{n}\left(X_{n}\right)\right)$ 
Note that the investment payoff $v$ is a function $f$ of the uncertainties,

$v\left(a_{j}\right)=f_{i}\left(X_{1}, \ldots, X_{n}\right)$

and the decision maker is assumed to choose the optimal alternative that maximizes the investment payoff. Without loss of generality, let $a_{0}$ be the optimal alternative under the base values of $X_{i}$ 's, $B=\left(\mu_{1}, \ldots, \mu_{n}\right)^{T}$.

The construction of the multivariate decision analysis model requires an initial assumption about the dependence relationships among the uncertainties, such as independence or assessed dependence measurements. For instance, when the dependence structure is modeled with normal copulas, which can be fully captured by the correlation matrix

$\Sigma_{i j}=\left(\rho_{i j}\right)_{i, j=1}^{n}$

the expected value of each alternative is a function of the correlations. Table 3 provides the correlation matrix for the Eagle Airlines example.

\section{Step 2 - Model Dependence using Copulas}

We next exploit the independence of the marginal distributions of the input variables under a copula structure to analyze the impact of the dependence structure through the following process:

1. Simulate independent variables $X_{1}, \ldots, X_{n}$ based on the given marginal distributions. As copulas separate the marginals and their dependence, we can simulate independent marginals using a Monte Carlo approach and include the dependence relationships in the following steps.

2. Find the independent uniform variables $u_{i}$ using the CDF for each of the simulated marginal variables: $u_{i}=F_{i}\left(X_{i}\right)$.

3. Construct the dependent uniform variables $u_{i}^{\prime}$. Since copulas are independent of the choice of the marginal distributions, we first specify the joint distribution of the underlying copula with uniform variables, and then combine it with the marginal distributions in the next step. Specifically, similar to the portfolio example, we recursively compute the dependent uniform variables 
$u_{i}^{\prime}=u_{i} \mid\left(u_{\sim i}=F_{i}\left(\mu_{\sim i}\right)\right)$,

where $u_{\sim i}$ denotes the vector of all factors but $u_{i}$, and $\mu_{\sim i}$ denotes the corresponding base value vector.

4. Point-to-point inverse marginal transformation. After the calculation of the dependent uniform variables $u_{i}^{\prime}$, we transform them to obtain the discrete approximations of the original uncertainties. The conditional $X_{i} \mid\left(X_{\sim i}=\mu_{\sim i}\right)$ are obtained by applying the inverse of the target marginal distribution function for each realization of $u_{i}^{\prime}$, $X_{i}=F_{i}^{-1}\left(u_{i}^{\prime}\right)$

For the Eagle Airlines example, following Clemen and Reilly (1999) and Wang and Dyer (2012), we use a normal copula as our modeling framework because its flexibility and analytical tractability fit the needs of the dependence modeling problem, and also because it facilitates the comparison of our results to the literature. As further justification for this approach, Montiel and Bickel $(2012,2013)$ show that a normal copula model provides a good representation of the joint distribution from a large collection of sampled joint distributions that match the assessed correlations. However, we also note that the choice of the copula is left to the modeler and this general framework could accommodate a t-copula or an Archimedean copula-based decision model which might, for example, better model tail dependence when extreme events are expected to coexist in an application.

A multivariate normal copula function $C_{N}$ is derived from a multivariate normal cumulative distribution function $\Phi_{\Sigma_{Z}}$ with mean zero and correlation matrix $\Sigma_{Z}$ by transforming the marginals by the inverse of the standard normal distribution function $\Phi$, given by:

$C_{N}\left(u_{1}, \ldots, u_{n}\right)=\Phi_{\Sigma_{Z}}\left(\Phi^{-1}\left(u_{1}\right), \Phi^{-1}\left(u_{2}\right), \ldots, \Phi^{-1}\left(u_{n}\right)\right)$, or equivalently,

$C_{N}\left(F_{1}\left(X_{1}\right), \ldots, F_{n}\left(X_{n}\right)\right)=\Phi_{\Sigma_{Z}}\left(\Phi^{-1}\left(F_{1}\left(X_{1}\right)\right), \ldots, \Phi^{-1}\left(F_{n}\left(X_{n}\right)\right)\right)$

The construction of the normal copula-based conditional uniform variable $u_{n}^{\prime}$ using the marginal and assessed correlations follows the procedure discussed in Wang and Dyer (2012):

$u_{n}^{\prime}=\Phi\left(A_{n 1} \Phi^{-1}\left(u_{1}\right)+\cdots+A_{n(n-1)} \Phi^{-1}\left(u_{n-1}\right)+A_{n(n)} \Phi^{-1}\left(u_{n}\right)\right)$ 
where $A_{i j}$ is the element of the Cholesky factorization that decomposes the covariance matrix $\Sigma$ as $\Sigma=A A^{T}$ to give the lower triangular matrix $A=\left(A_{i j}\right)_{i, j=1}^{n}$.

\section{Step 3 - Determine the Influential Variables}

As discussed in Step 2, all marginals are first independently simulated, with the dependence between variables incorporated via the unconditional and conditional uniform variables. Then, we perform a one-way sensitivity analysis on the model by varying the assumed outcome of one marginal and holding the other independent marginals constant at their base values. More specifically, a correlation-adjusted one-way sensitivity analysis measures the amount by which the objective function $v\left(a_{j}\right)$ changes or "swings" as the input variable is varied from its conditional low to its conditional high value, while all the other variables are fixed at their base values, as described in Step 2, reflecting the underlying dependence with the use of copulas. The notion that we can conduct a one-way sensitivity where only one variable is perturbed, yet still capture the dependence between variables is valid because all marginal distributions are independent in the copulas-framework.

To illustrate this step, we start with the independent marginal variables and work in a spreadsheet with a standard add-in such as @ Risk or Crystal Ball to implement the copulabased dependence analysis. Consistent with the assumption in Clemen and Reilly (2000) and Reilly (2000) and for the purpose of comparison, we model each marginal variable as a Triangular distribution with the low, mostly likely and high values given in Table 2. Using the

copula-based procedure yields the copula tornado diagram shown in Figure 2, in which we identify three influential variables: Price Level, Hours Flown and Operating Cost.

\subsection{A Comparison with the Standard and $n$-Way Sensitivity Analysis Approaches}

A standard one-way sensitivity analysis can be performed on $v=$ Profit - $\$ 4,200$ assuming all input variables are independent. The resulting tornado diagram shown in Reilly (2000) indicates that there are four input variables (Price Level, Hours Flown, Capacity and Operating Cost) that would be considered influential; i.e., they have the potential to change the decision policy. These variables each have possible realized values that could reduce the profit from Expand to a level below $\$ 4,200$, the point at which the function $v$ becomes negative, and therefore Money Market would be the recommended alternative. For example, holding all 
other variables at the base values and assuming independence, a decrease in the value of Capacity will eventually result in a negative value of $v$.

Table 2 reports the break-even decision-switching points for the nine input variables. For instance, the $47 \%$ break-even point for Capacity indicates that a small variation (3\%) from the base value will change the recommendation from Expand to Money Market. Thus, a variable can also be defined as influential if the break-even point is within the considered range of the variable. When the breakeven point for a variable is outside of this range, the variable is not considered influential. This is the case for the breakeven point for Percentage Financed at 97\% which is well above the upper bound of $50 \%$.

While this analysis is simple and easy to implement, a standard one-way sensitivity analysis relies on the independence assumption among the variables. Unfortunately, the independence assumption is often invalid in reality. For instance, it is reasonable to assume a negative correlation between the Price Level and the demand (Hours Flown and Capacity) according to basic microeconomics theory, and a positive correlation between Capacity and Operating Cost based on business practice.

To conduct an $n$-way probabilistic sensitivity analysis, many decision analysts rely on professional software applications for off-the shelf model building and for examining the sensitivity of dependent inputs in decision models. We will use the popular Excel Add-in @ RISK to illustrate this approach here. When there are correlations in the decision model, @ Risk first simulates independent random variables and then "adjusts the ranking and associating of samples within each iteration to yield the defined Spearman rank order correlation values". With the rank-adjusted simulated data that have the desired Spearman rank order correlations in Table 1, @Risk then calculates the output as a function of the correlated uncertainties. Next, it sorts each uncertainty into 10 bins in the default setting, and calculates the associated mean of the output in each bin for each uncertainty. Finally, @Risk reports the minimum and maximum of the 10 bins' outputs to draw the tornado chart to indicate a ranking of variables by sensitivity. The "Tornado - Change in Output Mean" sensitivity analysis conducted by@Risk is essentially an n-way sensitivity analysis in which all inputs are varied at the same time. 
There are several drawbacks to this approach. First, it is limited to the use of Spearman rank order correlations and is not designed for other dependence measures, such as Pearson product moment correlations, Kendall's Tau rank order correlations, or tail dependencies. Second, as it simulates all uncertainties simultaneously, the Tornado chart reflects the overall impact of all uncertainties and all correlations, and does not reveal the marginal impact of each uncertainty or correlation. As a result, some of the influential uncertainties and/or influential dependence relationships may be disguised by the joint influence of groups of the original variables, which can make it challenging for the decision analyst to interpret the results, and more importantly, to use them to design a parsimonious model. Third, it takes the assessed correlations as inputs and is not developed to examine the potential impact from correlation measurement errors.

For the Eagle Airline problem, the @ Risk sensitivity tornado output identifies only two influential variables (Hours Flown and Capacity) that could lead to a change in the recommended investment (Figure 3). These results are different from the results of our copulabased sensitivity analysis. The reason for these differences is the simultaneous modeling of all uncertainties in the @RISK approach, which means that the resulting sensitivities are joint, rather than marginal, as discussed above. Because the "Tornado - Change in Output Mean" sensitivity analysis provides joint sensitivities, it is also sensitive to the choice of the original group of variables. For instance, if we replace the distribution of the non-influential uncertainty Insurance with its deterministic base value and rerun this analysis, only Hours Flown remains as an influential variable.

\section{[Insert Figure 3 Here]}

To summarize these results, the standard sensitivity analysis approach would not provide results that reflect dependence relationships in the problem. The $n$-way provides allencompassing sensitivity results, albeit with a lack of information about marginal sensitivities for each variable.

\subsection{Discussion}

We notice some significant differences in the results from the standard independent and $n$-way analyses compared to those from the analyses discussed in Section 3.2 for the copula-based approach: (i) only three influential variables are identified in the copula-based approach 
compared to four from the standard sensitivity analysis (which also identifies Capacity as influential); (ii) while the ranking of variables in the tornado diagram of the standard sensitivity analysis indicated that Capacity caused the largest swing in the value of the objective function, followed by Price Level, Operating Cost, and Hours Flown, the ranking shown in Figure 2 is different, beginning with Price Level as the variable that caused the largest swing in the objective function value, followed by Hours Flown and Operating Cost; (iii) Capacity is not an influential variable, but it has the fourth longest bar and could become an influential variable when correlation assessment errors are included in the analysis; (iv) Charter Ratio is unlikely to be an influential variable, as it ranks in the bottom half of the list; (v) the order of Percentage Finance, Interest Rate and Purchase Price stay the same at the bottom of the tornado diagram and these are confirmed to be non-influential variables; and (vi) the worst case scenario in Figure 2 is associated with low Hours Flown (objective function value $\approx-\$ 5,000$ ), as compared to the worst case scenario in the standard sensitivity analysis, which is associated with low Capacity (objective function value $\approx-\$ 15,000$ ). The worst case scenarios for the other two influential variables are also significantly smaller in magnitude than the standard sensitivity analysis case.

The difference between the standard sensitivity analysis that assumes independence between the inputs and our proposed approach provides additional insights that are intuitively consistent with the inputs. For example, Capacity is positively correlated with Hours Flown (0.5) and Operating Cost (0.25), and also negatively correlated with Price Level (-0.25) and Charter Ratio (-0.25); therefore, when the capacity is low, the hours flown and the operating cost are also expected to be low, and at the same time, the price level is expected to be high. This can be viewed as a natural hedge embedded in the business and if it is not taken into consideration, the impact of Capacity is overstated. Once we take into account the hedge resulting from the dependence structure, the impact of Capacity no longer has as much impact on the Profit. Similarly, Price Level is negatively correlated with Hours Flown (-0.5) and Capacity (-0.25), and positively correlated with Charter Ratio (0.25), so when the price level is low, the hours flown and capacity are expected to increase which would offset the impact of a price reduction. As a result, the range of impact of Price Level considering the correlations is significantly narrower than the corresponding range of impact of Price Level indicated by the standard sensitivity analysis. 
Note that while we focus on the directional change sensitivity measure used in copula tornado diagrams in this paper for our illustration, our proposed framework can also apply the probabilistic sensitivity analysis measures to determine the influential variables in a straightforward manner. Our probabilistic sensitivity analysis measure can be obtained as a byproduct of the Monte Carlo simulation from step 2. For instance, with the simulated inputs and output, we can assess the correlation-based sensitivity analysis in Figure 4 (which also identifies the same most influential variables in the same order) to determine the influential inputs that would lead to the greatest expected modification in the output distribution.

[Insert Figure 4 Here]

\subsection{Determining the Influential Correlations and Extensions}

In addition to supporting a sensitivity analysis of the input variables, the copula approach can also be used to find the influential correlations whose values determine the recommended alternative within a reasonable range (e.g., the estimated range of error in the estimate of the correlation coefficient). If the correlations are assessed from historical data, there is a literature on the appropriate confidence intervals for Pearson's product moment correlations (Boomsma, 1977; Kraemer, 1980) and for rank order correlations such as Spearman's rank order correlations (Fieller et al., 1957; Choi, 1977; Iman and Conover 1982). If the correlations are assessed by an expert, Clemen and Reilly (1999) and Clemen et al. (2000) discuss different subjective correlation assessment methods for estimating the probability of concordance and conditional fractiles, and report that the average absolute error is 0.195 for experienced individuals and 0.254 for inexperienced individuals. These studies also find that even though the decision makers may not provide accurate point estimates for correlations, they are reasonably capable of providing upper and lower bounds for their estimates. This information can be used as a range of correlation variance for the sensitivity analysis of dependence, given the standard constraint that the correlation matrix is a valid correlation matrix (positive semidefinite).

As with the input variables, we define an influential correlation between variables as one which, when varied within specified limits, will lead to a change in the recommended alternative. This information can help the decision maker determine whether it is worthwhile to 
formally reassess $\rho_{i, j}$ to increase the accuracy of the estimated base value of $v$ and the associated decision policy.

We continue using the Eagle airlines example (Clemen and Reilly, 1999), and explore the range of possible perturbations of each non-zero correlation. Following Clemen et al. (2000) and Clemen and Reilly (1999), we perturb these correlations by \pm 0.25 , which is approximately the average mean absolute deviation for experts' assessment errors. When this change would lead to an invalid correlation matrix, we used the largest perturbation that would maintain a positive semi-definite correlation matrix. We then performed a one-way sensitivity analysis on each nonzero correlation using these lower and upper bounds while keeping the remaining correlations at their initial values.

Again, since copulas separate the marginal distributions and the dependence structure, the correlations are independent of the marginals. Thus, in this one-way sensitivity analysis, the independent marginals are kept constant at their base values, while the correlated marginals comove according to the perturbations of the correlations.

Assessing the impact of correlations with this one-way sensitivity analysis, it is clear that no correlations are influential when considered separately. The copula tornado diagram in Figure 5 shows that: (1) no perturbation of any single correlation will change the recommended alternative when the marginals are kept constant at their base values; (2) the correlation between Charter Ratio and Operating Cost has the largest impact on the outcome; (3) the correlation between two non-influential variables, Insurance and Percentage Finance, has the smallest impact; (4) the correlation between two influential variables, Hours and Price Level, has the second smallest impact, which indicates that the recommended alternative is robust to small perturbations of the correlation between these two variables; and (5) the correlations not shown, such as the correlation between Interest and Percentage Financed, are not ranked in the top ten and hence do not have significant impacts on the value of the alternative.

A tornado-diagram analysis of the correlations may provide considerable insights, although they are limited to impacts resulting from changing only one correlation at a time. We also note that although none of the correlations were influential in terms of affecting the decision policy in this example, the changes in correlation might be of interest if the analyst wishes to 
investigate the variance of a performance measure. In addition, we compute how much variation of each correlation input would be needed to lead to a different recommended alternative. For instance, the most influential correlation between Ratio and OpCost has to be changed from 0.25 to -0.48 to make the decision switch.

\section{[Insert Figure 5 Here]}

This method can be extended to an $n$-way sensitivity analysis to examine the combined impact of multiple uncertainties and/or correlations. For instance, the decision maker can vary each pair of correlations while keeping the remaining correlations constant: a two-way sensitivity. For example, if we want to examine the first two non-influential variables Capacity and Insurance, we might want to determine whether or not a perturbation of the related correlation and the range of the marginals could lead to a change in the recommended alternative. Capacity in particular was very close to being influential, so we want to know if variations in one of its correlations could cause it to become an influential variable.

We again use a Copula tornado diagram for the difference between expected profits for the two alternatives as an indicator of sensitivity. The output for a series of sensitivity analyses where two inputs are varied simultaneously is provided in Figure 6 and Figure 7. Each bar represents a different pair of inputs as specified in the y-axis labels. For instance, Figure 6 shows a series of sensitivity analyses between Capacity and its nonzero correlations, and between different pairs of Capacity's correlations with other uncertainties. We find that Capacity would become influential if its non-zero correlations are perturbed within reasonable ranges, but that all the combinations of two related non-zero correlations of Capacity are not influential.

[Insert Figure 6 Here]

Similarly, if we conduct sensitivity analysis for Insurance and its two non-zero correlations, the output shown in Figure 7 confirms that Insurance is not an influential variable. While not shown here, we also confirmed that Charter Ratio is not an influential variable with a two-way sensitivity analysis for Charter Ratio and its three related correlations.

[Insert Figure 7 Here] 


\section{Discussion and Conclusion}

The recommendations of decision models are sensitive to our assessments of inputs, including uncertainty and dependence. In general, sensitivity analysis is a source of guidance in modeling a decision problem, and it can also contribute to the specification of a model by assessing the individual contribution of a variable and determining whether to include it as a stochastic or deterministic input in the model. It also can provide context throughout the decision analysis process by providing information on the robustness of a model's recommendations, and it can help validate an economic model in the presence of uncertainty. Also, to the extent that the outcomes of a sensitivity analysis indicate the range of possible values for the objective function, users of the model can assess the upside and downside risks associated with alternative scenarios.

In comparison to the standard sensitivity analysis process, which mainly focuses on identifying the most influential individual uncertainties assuming independence, the copula approach also addresses sensitivity to dependence relationships between uncertainties. By using copulas to capture the dependence structure among uncertainties, we present a systematic and efficient procedure for performing sensitivity analysis to dependence in multivariate decision and risk analysis. In comparison to fully probabilistic, or $n$-way, sensitivity analysis techniques, our copula-based approach retains some of the practical advantages of standard one-way sensitivity analysis, especially the capability of isolating the marginal sensitivity to input variables, in an intuitive graphical interface while including the effect of dependencies.

We should note that there can be some disadvantages to using correlations to model dependencies among input variables. First, correlations are restricted in the type of relationship they can model. For example, Pearson's correlation assumes linearity and Spearman's correlation assumes monotonicity. Second, the correlation matrix must be positive definite otherwise it must be modified in some way (c.f. Rousseeuw and Molenberghs, 1994; Olkin, 1981; Lurie and Goldberg, 1998). Third, there are other dependence structures that may not be fully reflected by correlations. However, our proposed approach is flexible and does not rely on specific copula forms or correlations as the measure of dependence. As a result, this procedure can be used for a wide variety of problems (c.f. Chen et al. 2011; Mutha et al. 2015) 
to convey enhanced managerial insights not available through the standard sensitivity analysis when the optimal decision is sensitive to the dependence relationships.

In summary, the analysis of the Eagle Airlines example demonstrates how the copula-based sensitivity analysis enhances the existing methods. In particular, even when this method identifies the same set of influential variables, it conveys additional insights beyond the standard sensitivity methods. The copula approach provides decision analysts with a more complete description of the relative influence of variables and their correlations, information which can be helpful in risk analysis and in evaluating the range of possible scenarios. 


\section{References}

Ai J., P. L. Brockett, W. W. Cooper, and L. L. Golden, 2012, Enterprise Risk Management through Strategic Allocation of Capital, Journal of Risk and Insurance, 79 (1): 29-55.

Avramidis, A. N., N. Channouf, P. L'Ecuyer, 2009. Efficient Correlation Matching for Fitting Discrete Multivariate Distributions with Arbitrary Marginals and Normal-Copula Dependence. INFORMS J. Comput., 1(21): 88-106.

Baucells M., E. Borgonovo, 2013: Invariant Probabilistic Sensitivity Analysis, Management Science, 59 (11): 2536-2549. Bhattacharjya, D., R. Shachter, 2008. Sensitivity Analysis in Decision Circuits. In McAllester D., and Myllymaki P., editors, Proc. of 24th UAI, Finland, Helsinki AUAI Press, pp.32-42.

Bhattacharjya, D., R. Shachter, 2010. Three new sensitivity analysis methods for influence. Proceedings of the Twenty Sixth Conference on Uncertainty in Artificial Intelligence. Catalina Island, CA AUAI Press, 1-9.

Bhattacharjya, D., R. Shachter, 2012. Formulating Asymmetric Decision Problems as Decision Circuits. Decision Analysis, 9(2): 138-145.

Biller, B., 2009. Copula-Based Multivariate Input Models for Stochastic Simulation. Operations Research, 57(4): 878-892.

Bland, J. M., D. G. Altman, 1996. Measurement Error and Correlation Coefficients. BMJ: British Medical Journal, 313(7048): 41

Bornert, Michel, Fabrice Brémand, Pascal Doumalin, J-C. Dupré, Marina Fazzini, M. Grédiac, François Hild et al., 2009. Assessment of Digital Image Correlation Measurement Errors: Methodology and Results. Experimental Mechanics, 49(3): 353-370.

Boomsma, A., 1977. Comparing Approximations of Confidence Intervals for the Productmoment Correlation Coefficient. Statistica Neerlandica, 31(4): 179-185.

Borgonovo E. 2007. A New Uncertainty Importance Measure. Reliability Engineering and System Safety, 92: 771-784.

Borgonovo, E. 2013. Sensitivity Analysis in Decision Making. Wiley Encyclopedia of Operations Research and Management Science, 1-11.

Borgonovo, E., C. L. Smith, 2011. A study of interactions in the risk assessment of complex engineering systems: An application to space PSA. Operations research, 59(6): 14611476.

Borgonovo E., S. Tarantola, 2012. Advances in Sensitivity Analysis, Editorial for the Special Issue "Advances in Sensitivity Analysis", Reliability Engineering and System Safety, 107: $1-2$.

Borgonovo E., F. Tonoli, 2014. Decision-Network Polynomials and the Sensitivity of Decision Support Models, European Journal of Operational Research, 239(2), 490-503.

Camerer, C. and Weber, M., 1992. Recent developments in modeling preferences: Uncertainty and ambiguity. Journal of risk and uncertainty, 5(4): 325-370.

Campbell, J. E., G. R. Carmichael, T. Chai, M. Mena-Carrasco, Y. Tang, D. R. Blake, N. J. Blake et al., 2008. Photosynthetic control of atmospheric carbonyl sulfide during the growing season. Science, 322: 1085-1088.

Campolongo F., A. Saltelli, 1997. Sensitivity analysis of an environmental model: An application of different analysis methods. Reliability Engrg. System Safety, 57(1):49-69.

Chen, L., S. M. Gilbert, and Y. Xia, 2011. Private Labels: Facilitators or Impediments to Supply Chain Coordination. Decision Sciences, 42(3): 689-720.

Choi, S. C., 1977. Test of Equality of Dependent Correlations. Biometrika, 64 (3):645-647 
Clemen, R. T., T. Reilly, 1999. Correlations and Copulas for Decision and Risk Analysis. Management Sci., 45(2):208-224.

Clemen, R. T., T. Reilly., 2000. Making Hard Decision with DecisionTools, Duxbury Press, 2nd ed.

Clemen, R. T., G. W. Fisher, R. L. Winkler, 2000. Assessing Dependence: Some Experimental Results. Management Science, 46: 1100-1115.

Cherubini, U., E. Luciano, W. Vecchiato, 2004. Copula Methods in Finance, John Wiley \& Sons Ltd.

Doubilet, P., C. B. Begg, M. C. Weinstein, P. Braun, B. J. McNeil. 1985. Probabilistic sensitivity analysis using Monte Carlo simulation. Medical Decision Making 5(2): $157-$ 177.

Embrechts, P., F. Lindskog, A. McNeil, 1999. Modeling Dependence with Copulas and Applications to Risk Management. Department of Mathematics, ETHZ CH-8092 Zürich, Switzerland.

Etchart-Vincent, N., 2004. Is probability weighting sensitive to the magnitude of consequences? An experimental investigation on losses. Journal of Risk and Uncertainty, 28(3): 217-235.

Felli, J. C., G. B. Hazen, 1998. Sensitivity Analysis and the Expected Value of Perfect Information. Medical Decision Making, 18: 95-109.

Felli, J. C., G. B. Hazen, 2004. Javelin Diagrams: A Graphical Tool for Probabilistic Sensitivity Analysis. Decision Analysis, 1 (2): 93-107.

Fieller, E.C., H.O. Hartley, E. S. Pearson, 1957. Tests for Rank Correlation Coefficients. I. Biometrika, 44: 470-481

Fornell, C., D. F. Larcker, 1981. Evaluating Structural Equation Models with Unobservable Variables and Measurement Error. Journal of Marketing Research, 39-50.

Geir, N. V., L. Johnsen, S. Aanonsen, E. Vefring, 2003. Reservoir Monitoring and Continuous Model Updating using Ensemble Kalman Filter. In SPE Annual Technical Conference and Exhibition.

Hazen, G.B., M. Huang, 2006. Parametric Sensitivity Analysis Using Large-Sample Approximate Bayesian Posterior Distributions. Decision Analysis, 3(4): 208-219.

Hazen, G.B., 2014. Sensitivity Analysis via Information Density, 33(1):24-29.

He, Y., R.H. Huang, 2008. Risk Attributes Theory: Decision Making Under Risk. European Journal of Operational Research, 186(1): 243-260.

Helton, J., F.J. Davis, 2002. Illustration of Sampling-Based Methods for Uncertainty and Sensitivity Analysis. Risk Analysis, 22(3): 591-622.

Howard, R. A. 1983. The evolution of decision analysis. Howard RA, Matheson JE, eds. The Principles and Applications of Decision Analysis (Strategic Decisions Group, Menlo Park, CA), 5-16.

Iman, R. L., W. J. Conover, 1982. A distribution-free approach to inducing rank correlation among input variables. Communications in Statistics - Simulation and Computation 11(3): 311-334.

Iman R.L., S.C., Hora. 1990. A robust measure of uncertainty importance for use in fault tree system analysis. Risk Analysis 10(3):401-406.

Karni, E., 2009. A theory of medical decision making under uncertainty. Journal of Risk and Uncertainty, 39(1):1-16. 
Kraemer, H. C., 1980. Robustness of the Distribution Theory of the Product Moment Correlation Coefficient. Journal of Educational and Behavioral Statistics 5(2): 115-128.

Kousky, C., R. Cooke, 2009. The Unholy Trinity: Fat Tails, Tail Dependence, and MicroCorrelations. RFF Discussion Paper 09-36-REV.

Kucherenko, S., S. Tarantola, P. Annoni, 2012. Estimation of Global Sensitivity Indices for Models with Dependent Variables. Computer Physics Communications 183(4): 937-946.

Lin, P. S., C. W. Chang, C. H. Juang. 1999. Probabilistic sensitivity analysis for onedimensional solute transport. Civil Engrg. Environmental Systems, 17(1) 39-61.

Lord, J., M. A. Asante. 1999. Estimating uncertainty ranges for costs by the bootstrap procedure combined with probabilistic sensitivity analysis. Health Econom. 8(4) 323333.

Loomes, G. and Mehta, J., 2007. The sensitivity of subjective probability to time and elicitation method. Journal of risk and uncertainty, 34(3), 201-216.

Lowell, D. G., 1994. Sensitivity to Relevance in Decision Analysis. PhD dissertation.

Lurie, P. M., M. S. Goldberg, 1998. An Approximate Method for Sampling Correlated Random Variables from Partially-specified Distributions. Management Science, 44(2), 203-218.

Mara, T. A., S. Tarantola, 2012. Variance-based sensitivity indices for models with dependent inputs. Reliability Engineering \& System Safety, 107: 115-121.

Mutha, A., S. Bansal, D. Guide, 2015. Managing Demand Uncertainty through Core Acquisition in Remanufacturing. Production and Operations Management, Forthcoming.

Montiel, L.V., J.E. Bickel, 2012. A Simulation-Based Approach to Decision Making with Partial Information. Decision Analysis, 9(4) 329-347.

Montiel, L.V., J.E. Bickel, 2013. Approximating Joint Probability Distributions Given Partial Information. Decision Analysis, 10(1) 26-41.

Neilson, W.S., 2002. Comparative risk sensitivity with reference-dependent preferences. Journal of Risk and Uncertainty, 24(2): 131-142.

Nelsen, R., 2006, An Introduction to Copulas, 2nd ed., New York: NY: Springer.

Oakley, J., A. O'Hagan, 2004. Probabilistic Sensitivity Analysis of Complex Models: a \{Bayesian\} approach. Journal of the Royal Statistical Society, Series B, 66:751-769.

Oakley, J., 2009. Decision-theoretic Sensitivity Analysis for Complex Computer Models. Technometrics, 51(2): 121-129.

Office of Management and Budget, 2006. Proposed Risk Assessment Bulletin. https://obamawhitehouse.archives.gov/sites/default/files/omb/assets/omb/inforeg/propose d_risk_assessment_bulletin_010906.pdf.

Olkin, $\overline{\text { I. }}, 19 \overline{8} 1$. Range Restrictions for Product-moment Correlation Matrices. Psychometrika, 46(4), 469-472.

Reilly, T., 2000. Sensitivity Analysis for Dependent Variables. Decision Sciences, 31(3), 551572.

Rousseeuw, P. J., G. Molenberghs, 1994. The Shape of Correlation Matrices. The American Statistician, 48(4), 276-279.

Saltelli, A., A. Paola, 2010. How to avoid a perfunctory sensitivity analysis. Environmental Modelling \& Software, 25: 1508-1517.

Saltelli A., J. Marivoet, 1990. Non-parametric statistics in sensitivity analysis for model output: A comparison of selected techniques. Reliability Engrg. System Safety. 28(2):229-253. 
Saltelli, A., M. Ratto, S. Tarantola, F. Campolongo, 2012. Update 1 of: Sensitivity Analysis for Chemical Models. Chemical Reviews. 112: 1-21.

Saltelli, Andrea, P. Annoni, 2010. How to avoid a perfunctory sensitivity analysis. Environmental Modelling \& Software 25: 1508-1517.

Sarin, R. K., 1978. Elicitation of Subjective Probabilities in the Context of Decision-making. Decision Sciences, 9(1), 37-48.

Sklar, A., 1959. Fonctions de répartition a n dimensions et leurs marges, Publ. Inst. Statist. Univ. Paris 8: 229-231.

Strong, M., J. Oakley, 2013. An Efficient Method for Computing Partial Expected Value of Perfect Information for Correlated Inputs. Medical Decision-Making, 33: 755-766.

Storlie, C.B. et al., 2009. Implementation and evaluation of nonparametric regression procedures for sensitivity analysis of computationally demanding models. Reliability Engineering \& System Safety, 94(11):1735-1763.

Van der Gaag, L.C., S. Renooij, V.M.H. Coupe, 2007. Sensitivity Analysis of Probabilistic Networks. Advances in Probabilistic Graphical Models, Studies in Fuzziness and Soft Computing. 214:103-124.

Wagner, H.M., 1995. Global Sensitivity Analysis. Operations Research, 43(6): 948-969.

Wang, T., J. Dyer, 2012. A Copulas-based Approach to Modeling Dependence in Decision Trees. Operations Research, 60 225-242.

Wang, T., J. S. Dyer, and J. C. Butler, 2015. Modeling Correlated Discrete Uncertainties in Event Trees with Copulas, Risk Analysis, 36(2), 396-410.

Wang, T., J. S. Dyer, and W. J. Hahn, 2015. A Copula-based Approach for Generating Lattices. Review of Derivatives Research, 18(3), 263-289.

Wei, P., Z. Lu, J. Song, 2014. Moment-independent sensitivity analysis using copula. Risk Analysis, 34(2): 210-222. 


\section{Table 1: Results of sensitivity analyses $(\rho=0.9)$}

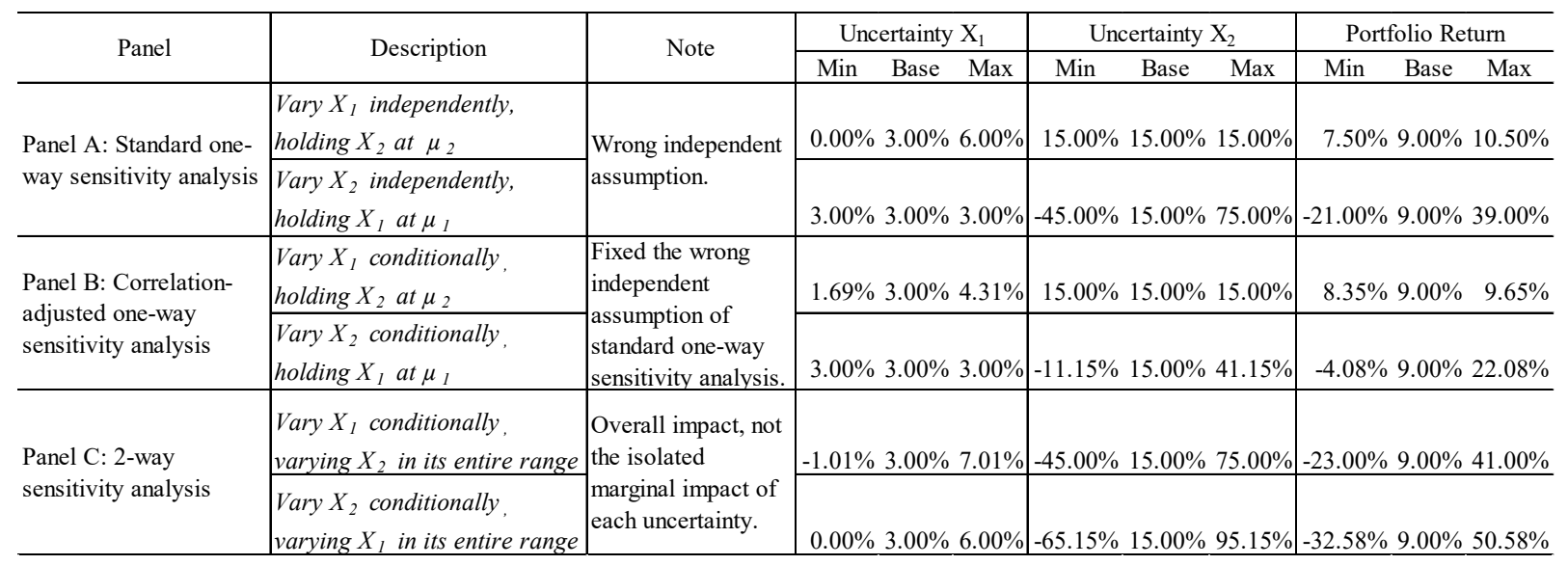


Table 2: Range of Input Variables and Estimated Standard Deviation

\begin{tabular}{lrrrrr}
\hline Variable $X i$ & Low & Base & High & Estimated SD StDev & Break Even Point \\
& & & & & \\
\hline Charter Ratio & $45 \%$ & $50 \%$ & $70 \%$ & $9.26 \%$ & $40 \%$ \\
Capacity & $40 \%$ & $50 \%$ & $60 \%$ & $7.41 \%$ & $47 \%$ \\
Price Level & $\$ 95$ & $\$ 100$ & $\$ 108$ & $\$ 4.81$ & $\$ 97$ \\
Hours Flown & 500 & 800 & 1000 & 185 & 664 \\
Operating & $\$ 230$ & $\$ 245$ & $\$ 260$ & $\$ 11.11$ & $\$ 252$ \\
Percentage Financed & $30 \%$ & $40 \%$ & $50 \%$ & $7.41 \%$ & $97 \%$ \\
Interest Rate & $10.50 \%$ & $11.50 \%$ & $13 \%$ & $0.93 \%$ & $28.00 \%$ \\
Insurance & $\$ 18,00$ & $\$ 20,00$ & $\$ 25,00$ & $\$ 2,593$ & $\$ 25,77$ \\
Purchase Price & $\$ 85,00$ & $\$ 87,50$ & $\$ 90,00$ & $\$ 1,852$ & $\$ 213,04$ \\
\hline Source: Reilly (2000) & & & &
\end{tabular}


Table 3: Correlation Matrix for Eagle Airlines

\begin{tabular}{llllllllll}
\hline & Hours & PriceL & Cap & Ratio & OpCost & Insur & \%Fin & Inter & PurPr \\
Hours & 1 & & & & & & & & \\
PriceL & -0.5 & 1 & & & & & & & \\
Cap & 0.5 & -0.25 & 1 & & & & & & \\
Ratio & 0.25 & 0.25 & -0.25 & 1 & & & & & \\
OpCost & 0 & 0 & 0.25 & 0.25 & 1 & & & & \\
Insur & 0.25 & 0 & 0 & 0 & 0 & 1 & & & \\
\%Fin & 0 & 0 & 0 & 0 & 0.25 & 0.25 & 1 & & \\
Inter & 0 & 0 & 0 & 0 & 0 & 0 & -0.5 & 1 & \\
PurPr & 0 & 0 & 0 & 0 & 0 & 0 & 0.75 & -0.25 & 1 \\
\hline
\end{tabular}


Figure 1 - Tornado Diagram for Different Sensitivity Methods

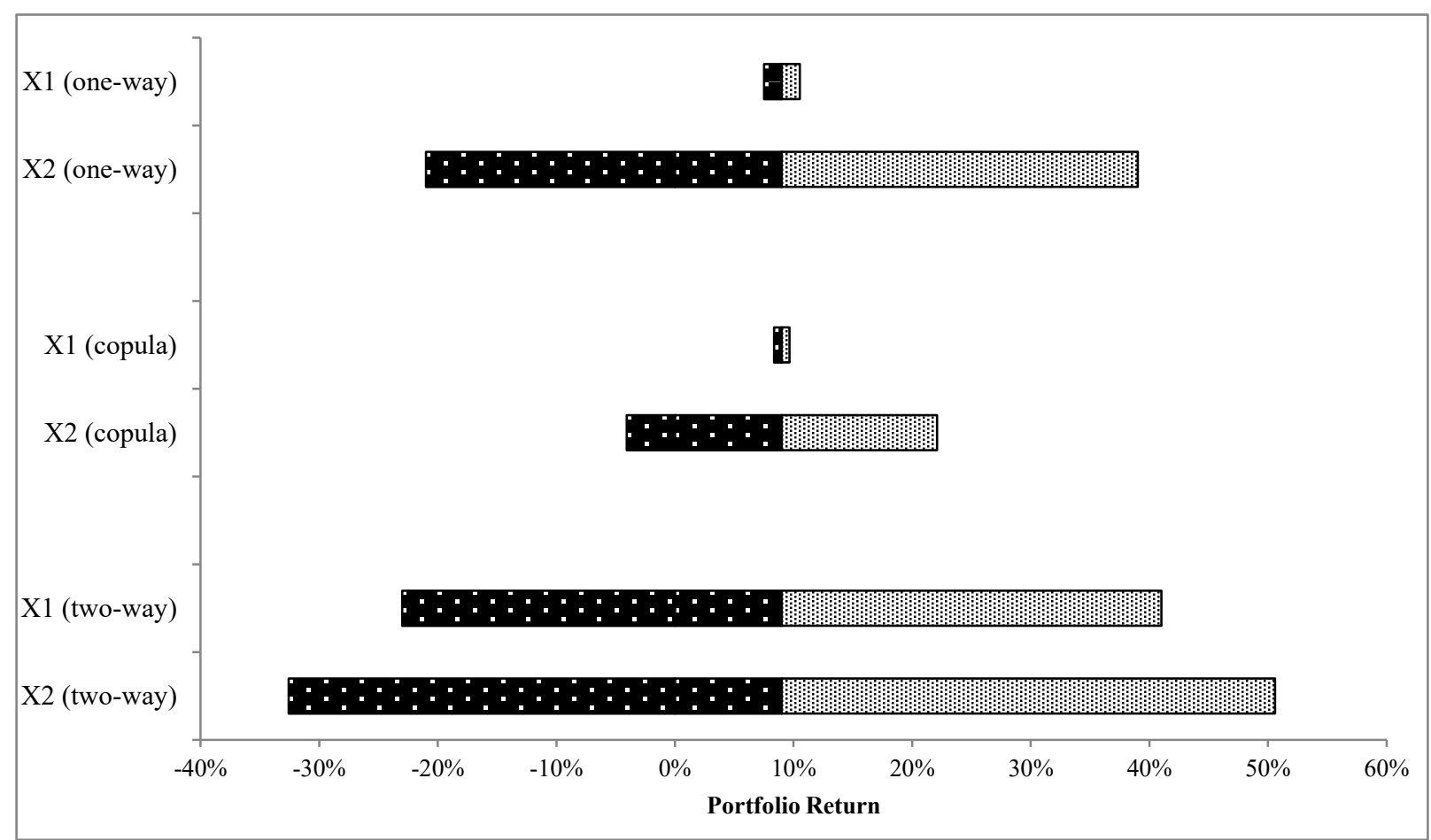


Figure 2: Copula Tornado Diagram: One-way Sensitivity for Uncertainties, including Dependence

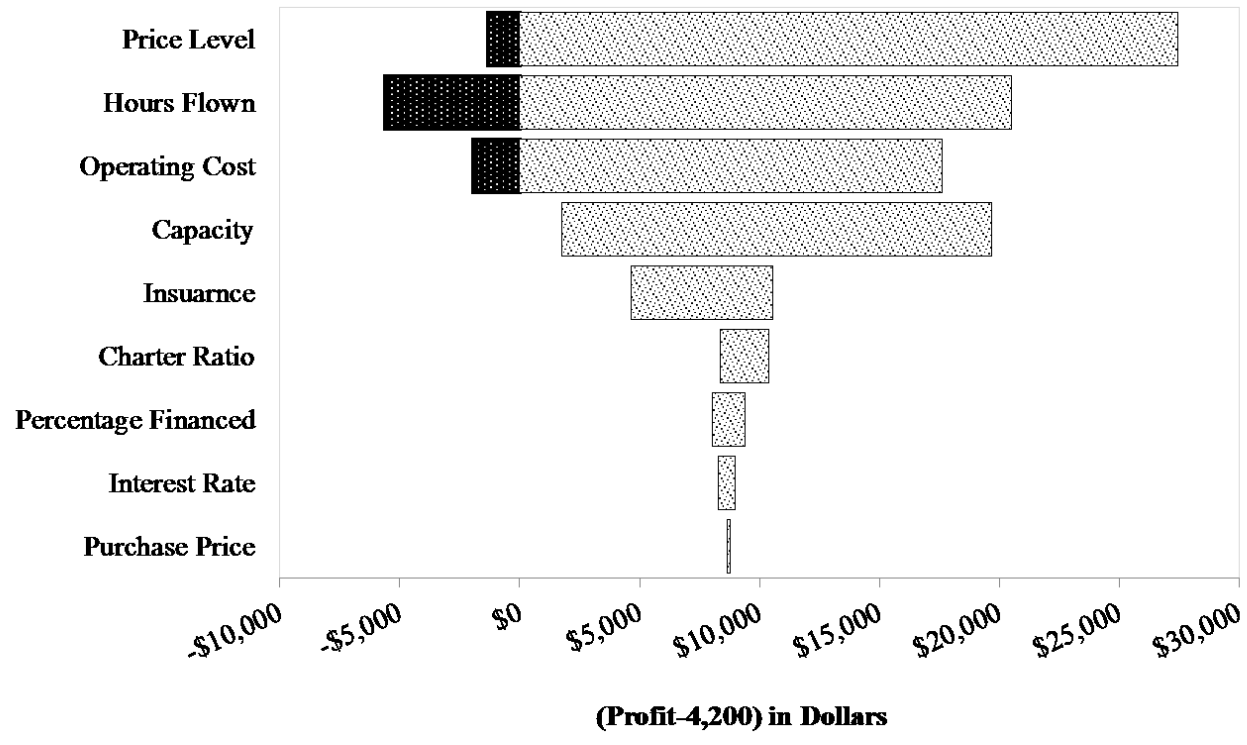


Figure 3: Tornado Chart from @RISK for Eagle Airlines Problem

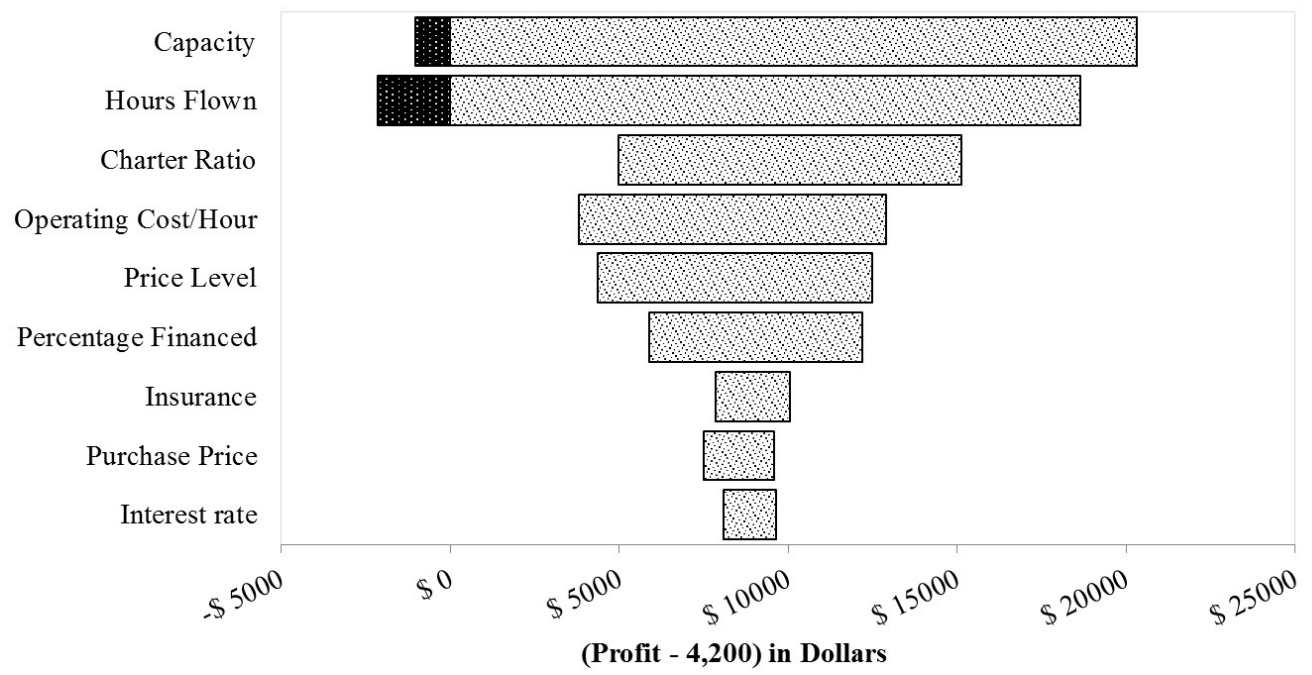


Figure 4: Rank Order Correlation Chart for Eagle Airlines Problem

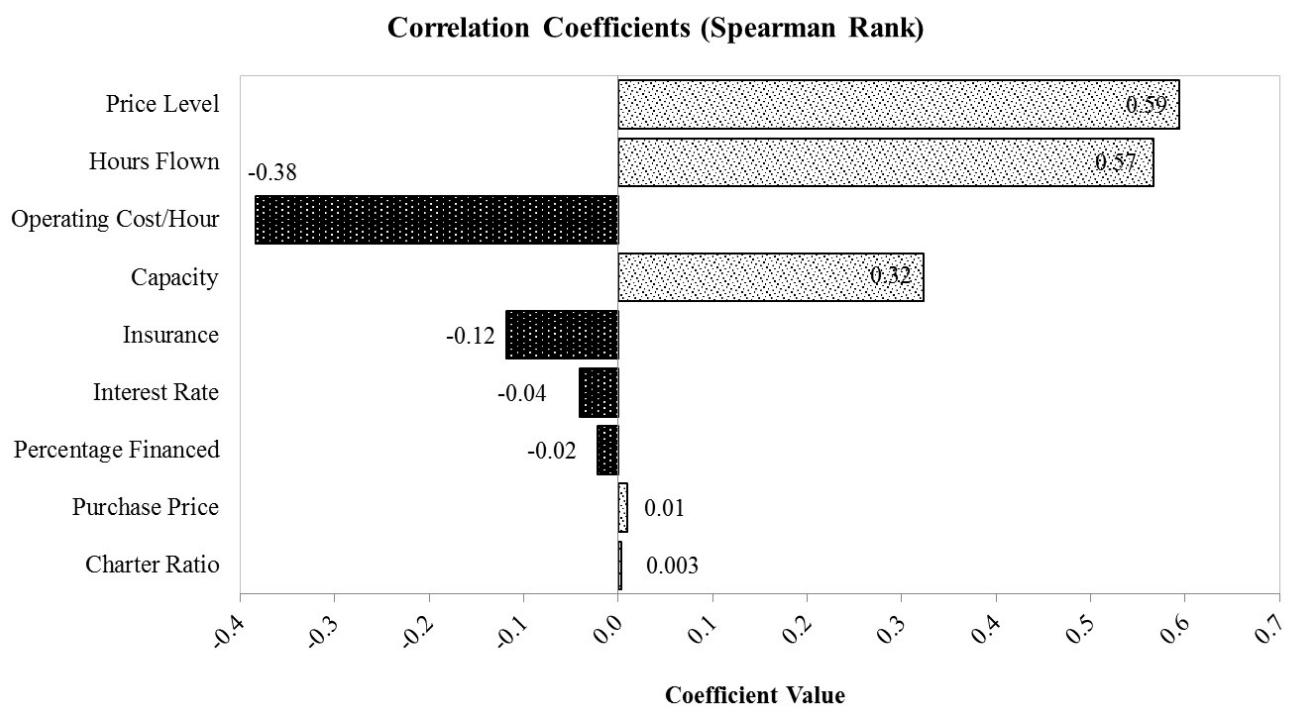


Figure 5: Copula Tornado Diagram: One-way Sensitivity Analysis for Correlations

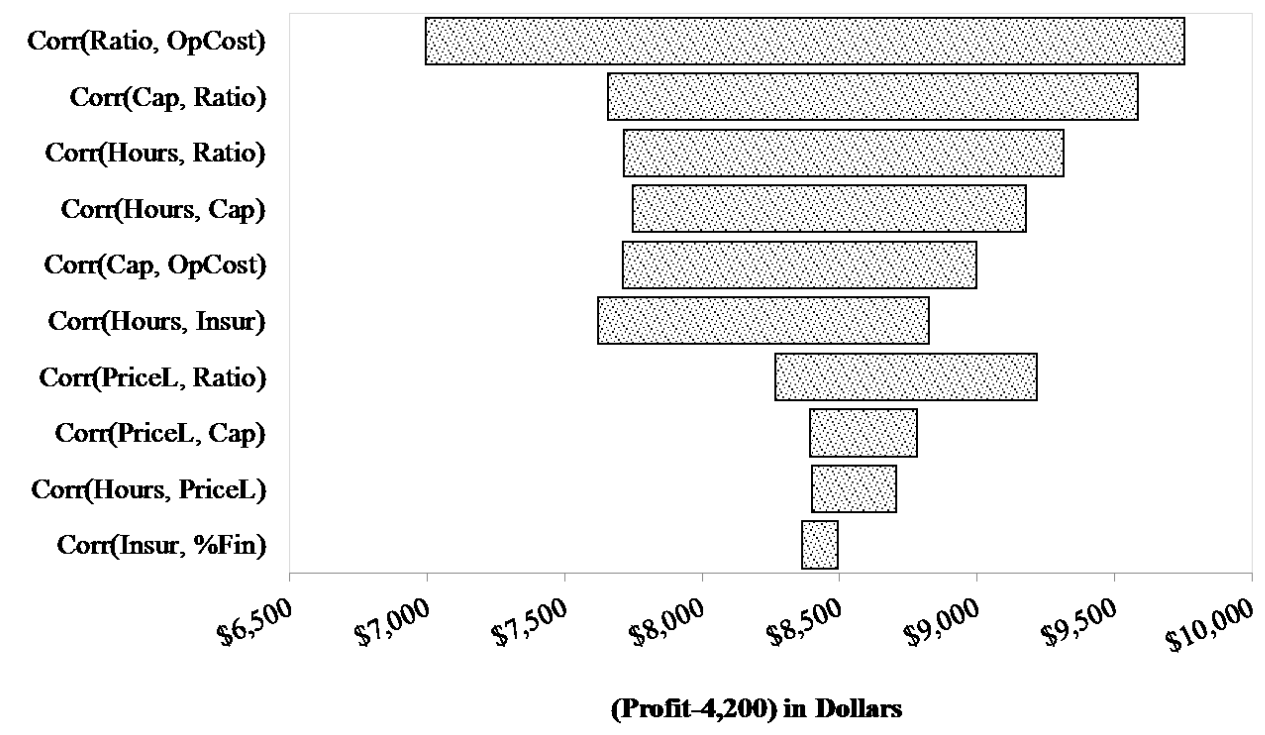


Figure 6: Copula Tornado Diagram: Sensitivity Analysis for Capital and its Related Correlations

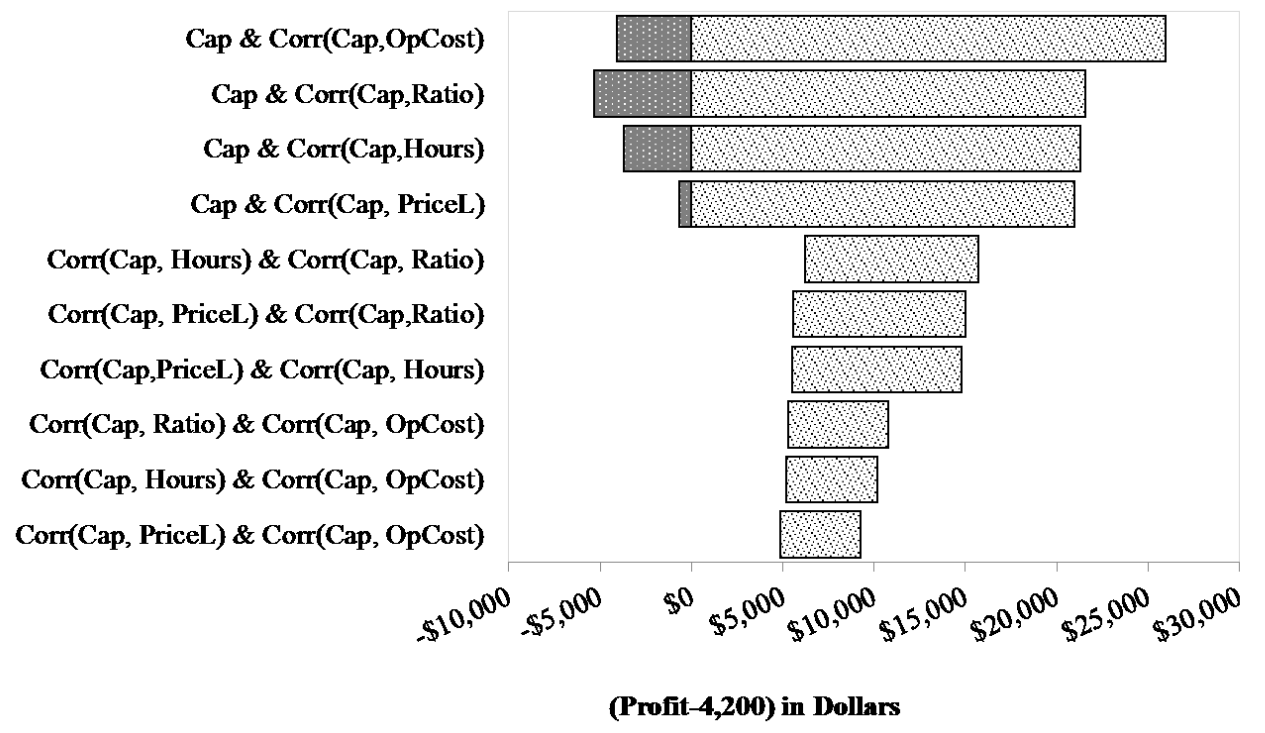


Figure 7: Copula Tornado Diagram: Sensitivity Analysis for Insurance and its Related Correlations

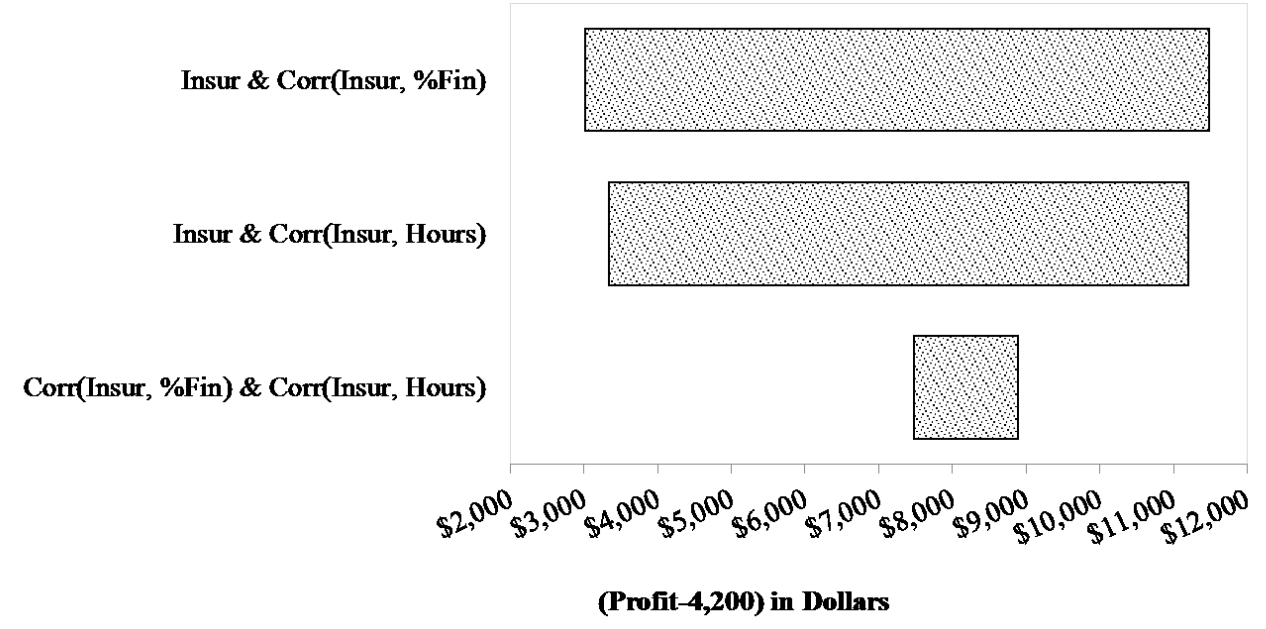

